\title{
Allopurinol Enhanced Adenine Nucleotide Repletion after Myocardial Ischemia in the Isolated Rat Heart
}

Robert D. Lasley, ${ }^{\star}$ Stephen W. Ely, ${ }^{\star *}$ Robert M. Berne, ${ }^{\star}$ and Robert M. Mentzer, Jr.**

${ }^{*}$ Departments of Physiology and ${ }^{\ddagger}$ Surgery, University of Virginia School of Medicine, Charlottesville, Virginia 22908

\begin{abstract}
Allopurinol, a competitive inhibitor of xanthine oxidase, has been shown to have a protective effect on ischemic myocardium, but its mechanism of action remains controversial. We used an isolated rat heart preparation to test the hypothesis that allopurinol could restore adenosine triphosphate (ATP) levels and improve the recovery of left ventricular function after global myocardial ischemia. Hearts were equilibrated for $30 \mathrm{~min}$, subjected to $10 \mathrm{~min}$ of global, normothermic $\left(37^{\circ} \mathrm{C}\right)$ ischemia, and reperfused for 15,30 , and $60 \mathrm{~min}$. Hearts treated with allopurinol $(100 \mu \mathrm{M})$ exhibited greater ATP levels and improved function during reperfusion than did untreated control hearts. Hearts treated with hypoxanthine $(100 \mu \mathrm{M})$, the substrate for xanthine oxidase, also showed increased ATP and functional recovery compared with controls. These results suggest that allopurinol may protect the globally ischemic myocardium by enhancing the salvage of hypoxanthine for reincorporation into adenine nucleotides.
\end{abstract}

\section{Introduction}

Reperfusion of ischemic myocardium is characterized by depressed contractile function and an increased susceptibility to ventricular arrhythmias. Several studies have suggested that these abnormalities are due to the depletion of high energy phosphates and the rapid catabolism and washout of the adenine nucleotide precursors adenosine, inosine, and hypoxanthine (1-5). Recently there have been many reports implicating oxygen free radicals as the mediator of reperfusion injury (6-10). Oxygen free radicals can be generated by the oxidation of catecholamines by monoamine oxidase, the action of phospholipases on membrane phospholipids, superoxide leak in the mitochondrial electron transport chain, NADPH oxidase activity in leukocytes, and by the action of xanthine oxidase (11).

Xanthine oxidase, which catalyzes the catabolism of hypoxanthine and xanthine to uric acid, is believed to be the major source of oxygen free radicals in the ischemic myocardium $(11,12)$. Initial studies with allopurinol, a competitive inhibitor of xanthine oxidase, suggested that its protective effect on ischemic myocardium was due to the preservation of purine bases for the resynthesis of ATP (13-17). More recent investi-

Address reprint requests to Dr. Lasley, Department of Surgery, Erie County Medical Center, Room CC 108, Clinical Center Building, 462 Grider Street, Buffalo, NY 14215.

Received for publication 10 February 1987 and in revised form 15 June 1987.

J. Clin. Invest.

(c) The American Society for Clinical Investigation, Inc. $0021-9738 / 88 / 01 / 0016 / 05 \$ 2.00$

Volume 81, January 1988, 16-20 gations have suggested that the beneficial effects are due to the inhibition of oxygen free radical formation (6-8).

We tested the hypothesis that allopurinol protects the globally ischemic myocardium by enhancing the repletion of high energy phosphates. In addition, we supplied exogenous hypoxanthine, a substrate for xanthine oxidase, to determine whether its presence would exacerbate tissue injury during reperfusion by stimulating the formation of oxygen free radicals or enhance recovery by providing substrate for the purine salvage pathway.

\section{Methods}

Male Wistar rats (250-300 g) were heparinized (500 U i.p.) and anesthetized with halothane. The hearts were rapidly excised and immediately placed into ice-cold Krebs-Henseleit buffer and then mounted by the aortic root on a Langendorff isolated heart apparatus. The hearts were perfused with a modified Krebs-Henseleit buffer consisting of (in millimoles per liter): $\mathrm{NaCl} 117, \mathrm{KCl} 4.7, \mathrm{MgSO}_{4} 1.2, \mathrm{KH}_{2} \mathrm{PO}_{4}$ 1.2, $\mathrm{CaCl}_{2}$ 3.0, EDTA 0.5, $\mathrm{NaHCO}_{3}$ 23.7, glucose 11.5, and pyruvate 1.0. The perfusate was gassed with $95 \% \mathrm{O}_{2} / 5 \% \mathrm{CO}_{2}, \mathrm{pH}$ adjusted to 7.40 , and maintained at $37^{\circ} \mathrm{C}$ by a constant temperature reservoir.

Hearts were perfused at a constant flow of $21-22 \mathrm{ml} / \mathrm{min}$ with a perfusion pump (model 1215; Harvard Apparatus Co., S. Natick, MA). This flow rate produced a coronary perfusion pressure of $\sim 100$ $\mathrm{mmHg}$ as measured from a side arm of the aortic cannula. A salinefilled latex balloon connected via a polyethylene catheter to a (1200C; Hewlett-Packard Co., Palo Alto, CA) pressure transducer was inserted into the left ventricle via the left atrium and inflated to yield an end diastolic pressure of $0 \mathrm{mmHg}$. Left ventricular developed pressure, heart rate, and coronary perfusion pressure measurements were recorded with a preamplifier (H-P 7809A; Hewlett-Packard Co.) and a chart recorder (2M3C; MFE Corp., Salem, NH). Coronary flow rate was determined from timed collections of effluent overflow from a heart bath filled with perfusate and maintained at $37^{\circ} \mathrm{C}$.

Experimental protocol. Hearts were assigned to one of three groups: untreated, allopurinol treated, or hypoxanthine treated. The treated hearts were perfused with $100 \mu \mathrm{M}$ allopurinol or $100 \mu \mathrm{M}$ hypoxanthine throughout the entire perfusion period. Hearts that could not maintain a spontaneous ventricular rate of $260-280 \mathrm{bpm}$ or that developed prolonged arrhythmias were discarded.

The hearts were perfused for a 30 -min equilibration period and then subjected to $10 \mathrm{~min}$ of global, normothermic ischemia. Reperfusion was initiated by gradually returning flow to the preischemic rate over a period of $3 \mathrm{~min}$. Coronary flow rate, coronary perfusion pressure, and left ventricular developed pressure were recorded at the end of $30 \mathrm{~min}$ equilibration and at 15,30 , and $60 \mathrm{~min}$ of reperfusion. At the end of each time period, the hearts were freeze-clamped in Wollenberger tongs precooled to the temperature of liquid nitrogen $\left(-80^{\circ} \mathrm{C}\right)$ for the determination of adenine nucleotide content. The tissue samples were stored under liquid nitrogen until the assay for adenine nucleotides was performed.

The release of nucleosides was determined by the timed collections of coronary effluent at the end of 30 min equilibration, and at $0.5,1$, $1.5,3,6$, and $10 \mathrm{~min}$ of reperfusion. The effluent samples were air dried, resuspended in $100 \mathrm{mM} \mathrm{KH}_{2} \mathrm{PO}_{4}, \mathrm{pH} \mathrm{4.0,} \mathrm{and} \mathrm{frozen} \mathrm{at}-80^{\circ} \mathrm{C}$ until assayed for nucleoside content. 
Tissue and perfusate analysis. 10-15-mg samples of frozen tissue were rapidly weighed to the nearest $0.05 \mathrm{mg}$, immediately transferred to homogenizing tubes (Radnoti Econogrind Homogenizer; A. H. Thomas Co., Philadelphia, PA) containing $0.4 \mathrm{ml}$ of ice-cold $12 \%$ TCA and extracted for 30 min with periodic grinding to produce a homogenous slurry. The suspension was centrifuged at $4^{\circ} \mathrm{C}$ for $3 \mathrm{~min}$ at 1,000 $g$, after which the supernatant fraction was neutralized with $1.2 \mathrm{ml}$ of $0.5 \mathrm{M}$ tri- $n$-octylamine in freon. The mixture was vigorously mixed for $1 \mathrm{~min}$, centrifuged at $4^{\circ} \mathrm{C}$ for $3 \mathrm{~min}$ at $1,000 \mathrm{~g}$, and the aqueous layer was removed and stored at $-80^{\circ} \mathrm{C}$ until analysis by HPLC. Separate portions of frozen tissue were dried for $24 \mathrm{~h}$ at $100^{\circ} \mathrm{C}$ to determine dry weight.

A $100-\mu 1$ sample of the tissue extract was injected onto a strong anion exchange column $(25 \mathrm{~cm} \times 0.46 \mathrm{~cm}, 10 \mu \mathrm{m}$ particle size; SAX; Whatman, Inc., Clifton, NJ) with a Waters model 720 system controller and M45 and 6000A solvent delivery systems (Waters Associates, Inc., Milford, MA). The adenine nucleotides were eluted using a linear gradient of $100 \%$ buffer $\mathrm{A}\left(5 \mathrm{mM} \mathrm{NH}_{4} \mathrm{H}_{2} \mathrm{PO}_{4}, \mathrm{pH} 2.8\right)$ to $100 \%$ buffer $\mathrm{B}\left(750 \mathrm{mM} \mathrm{NH}_{4} \mathrm{H}_{2} \mathrm{PO}_{4}, \mathrm{pH} 3.8\right)$ established over a period of 36 min at a flow rate of $2 \mathrm{ml} / \mathrm{min}$. A 14-min period was allowed for column reequilibration. AMP, ADP, and ATP were determined by absorbance at $254 \mathrm{~nm}$ with an absorbance detector (model 440, Waters Associates, Inc.). Peaks were identified by comparison of retention times to those of known external standards and peak quantification was determined by peak height. Myocardial nucleotide content was expressed as micromoles per gram dry weight of tissue.

The nucleoside content of the coronary effluent was determined by

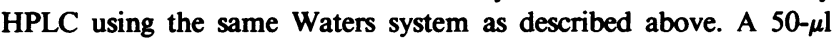
sample of the resuspended effluent was injected onto an Altex Ultrasphere ODS 18 column (Beckman Instruments, Inc., Fullerton, CA) $(25 \mathrm{~cm} \times 0.46 \mathrm{~cm}, 5 \mu \mathrm{m}$ particle size) and eluted with buffer $A(100$ $\left.\mathrm{mM} \mathrm{KH} \mathrm{PO}_{4}, \mathrm{pH} 4.0\right)$ and buffer $\mathrm{B}\left(100 \mathrm{mM} \mathrm{KH}_{2} \mathrm{PO}_{4}, 25 \%\right.$ methanol, pH 5.13) at a flow rate of $1 \mathrm{ml} / \mathrm{min}$. After an initial $3 \mathrm{~min}$ of $100 \%$ buffer A, a linear gradient to $90 \%$ buffer B was established over the next $32 \mathrm{~min}$. The column was reequilibrated for $13 \mathrm{~min}$. Uric acid, hypoxanthine, xanthine, inosine, and adenosine were identified by retention times to known external standards and quantified by peak height. Nucleoside release was expressed as nanomoles per minute.

Statistical analysis. Values are reported as means plus or minus standard errors of the mean (SE). Allopurinol and hypoxanthinetreated hearts were compared to control hearts using the nonpaired Student's $t$ test.

\section{Results}

Table I shows the results of allopurinol and hypoxanthine treatment on tissue adenine nucleotide content during ischemia and reperfusion. The data indicate that allopurinol significantly increased ATP content at 30 and $60 \mathrm{~min}$ of reperfusion. ADP content was increased at the end of $10 \mathrm{~min}$ of ischemia and at 15 and $60 \mathrm{~min}$ of reperfusion. AMP content was also augmented by allopurinol treatment at the end of 10 min of ischemia. Total adenine nucleotides (TAN) ${ }^{1}$ were significantly increased after $10 \mathrm{~min}$ of ischemia and after 15 and $60 \mathrm{~min}$ of reperfusion.

Hypoxanthine treatment increased ATP levels at $30 \mathrm{~min}$ of reperfusion and augmented ADP content at 30 and $60 \mathrm{~min}$ of reperfusion. TAN were also elevated at $30 \mathrm{~min}$ of reperfusion. Neither hypoxanthine nor allopurinol had any effect on adenine nucleotide content at the end of the 30 -min equilibration period.

Fig. 1 illustrates the effects of allopurinol and hypoxanthine on the recovery of left ventricular function (expressed as

1. Abbreviations used in this paper: TAN, total adenine nucleotide. the percentage of the developed pressure at the end of $30 \mathrm{~min}$ of equilibration). There were no differences in left ventricular developed pressures at the end of equilibration in the control, allopurinol, and hypoxanthine groups $(99 \pm 3,90 \pm 4,87 \pm 3$ $\mathrm{mmHg}$, respectively). Control hearts recovered to $76 \pm 5 \%$, $74 \pm 5 \%$, and $88 \pm 2 \%$ of the 30 -min equilibration values at 15 , 30 , and $60 \mathrm{~min}$ of reperfusion, respectively. Allopurinol treatment resulted in a significantly greater return of function to $91 \pm 3 \%, 90 \pm 4 \%$, and $97 \pm 1 \%$ of equilibration developed pressures at 15, 30, and $60 \mathrm{~min}$ of reperfusion, respectively. Hypoxanthine treatment resulted in a significantly greater return of function $(88 \pm 2 \%)$ at $30 \mathrm{~min}$ of reperfusion compared to the control group.

The effect of allopurinol and hypoxanthine on the release of xanthine and uric acid is depicted in Fig. 2. Allopurinol treatment resulted in a marked decrease in the release of xanthine and uric acid at $0.5,1.5,3$, and $6 \mathrm{~min}$ of reperfusion $(10.8 \pm 1.9,5.7 \pm 0.7,5.3 \pm 0.7$, and $3.8 \pm 0.5 \mathrm{nmol} / \mathrm{min}$, respectively) compared to the untreated hearts $(66.8 \pm 10.0,53.2 \pm 9.6$, $62.3 \pm 9.9$, and $25.8 \pm 9.0 \mathrm{nmol} / \mathrm{min}$, respectively). In fact, the release of xanthine plus uric acid in the allopurinol group was entirely in the form of xanthine; no uric acid was detected. Neither xanthine nor uric acid was detected at $10 \mathrm{~min}$ of reperfusion in the allopurinol-treated hearts. Allopurinol treatment had no effect on the release of adenosine and inosine during reperfusion. Hypoxanthine treatment resulted in a marked increase in the release of xanthine and uric acid at 0.5 , $1.5,3,6$, and $10 \mathrm{~min}$ of reperfusion $(133.6 \pm 15.8,99.2 \pm 7.7$, $109.0 \pm 7.7,107.4 \pm 10.4$, and $92.6 \pm 5.6 \mathrm{nmol} / \mathrm{min}$, respectively). These results indicate that xanthine oxidase is present in the myocardium and that it is effectively inhibited by allopurinol.

Fig. 3 illustrates the effect of allopurinol on the release of hypoxanthine. There was a significantly greater release of hypoxanthine from the allopurinol-treated hearts at $0.5,1.5,3$, and $6 \mathrm{~min}$ of reperfusion $(93.8 \pm 9.0,62.7 \pm 2.7,53.2 \pm 4.8$, and $16.3 \pm 3.5 \mathrm{nmol} / \mathrm{min}$, respectively) compared to the control hearts $(36.6 \pm 6.5,22.3 \pm 2.5,19.0 \pm 1.7$, and $5.4 \pm 1.5 \mathrm{nmol} / \mathrm{min}$, respectively). These results demonstrate that allopurinol prevents the catabolism of hypoxanthine to xanthine and uric acid.

\section{Discussion}

The results of this study confirm that allopurinol has a protective effect on globally ischemic myocardium as evidenced by the greater functional recovery throughout the reperfusion period. Allopurinol treatment resulted in significantly greater levels of ATP and total adenine nucleotides (ATP + ADP + AMP) during reperfusion, suggesting that allopurinol's beneficial effects may be due to enhanced repletion of high energy phosphates. Supplementation of the perfusion medium with hypoxanthine, the substrate for xanthine oxidase, maintained function and replenished ATP content to levels similar to that observed with allopurinol, rather than depressing functional recovery as would be predicted from the observed increase in xanthine oxidase activity and the concomitant production of oxygen free radicals. These results suggest that allopurinol's beneficial effects on globally ischemic myocardium may be due to the enhancement of purine salvage rather than the prevention of oxygen free radical formation. 
Table I. Myocardial Adenine Nucleotide Contents during Ischemia and Reperfusion

\begin{tabular}{|c|c|c|c|c|c|c|c|c|c|c|c|c|}
\hline & \multicolumn{3}{|l|}{ ATP } & \multicolumn{3}{|l|}{ ADP } & \multicolumn{3}{|l|}{ AMP } & \multicolumn{3}{|l|}{ TAN } \\
\hline & $\mathrm{C}$ & ALLO & HYPO & C & ALLO & HYPO & C & ALLO & HYPO & $\mathrm{C}$ & ALLO & HYPO \\
\hline \multirow[t]{2}{*}{$30^{\prime}$ Equil } & 23.85 & 23.33 & 22.52 & 8.40 & 8.35 & 6.02 & 1.16 & 1.00 & 0.421 & 33.41 & 32.71 & 28.96 \\
\hline & \pm 1.38 & \pm 0.74 & \pm 1.51 & \pm 1.22 & \pm 0.48 & \pm 0.37 & \pm 0.42 & \pm 0.09 & \pm .02 & \pm 2.33 & \pm 1.26 & \pm 1.88 \\
\hline \multirow[t]{2}{*}{$10^{\prime}$ Ischemia } & 8.85 & 11.18 & 9.66 & 11.44 & $16.56^{*}$ & 11.71 & 3.56 & $6.25^{*}$ & 4.10 & 23.85 & $33.98^{*}$ & 25.47 \\
\hline & \pm 0.99 & \pm 0.52 & \pm 1.04 & \pm 0.91 & \pm 0.99 & \pm 1.06 & \pm 0.50 & \pm 0.62 & \pm 0.71 & \pm 1.83 & \pm 1.09 & \pm 1.20 \\
\hline \multirow[t]{2}{*}{$15^{\prime} \mathrm{RP}$} & 15.21 & 16.62 & 16.63 & 4.36 & $6.22^{*}$ & 5.07 & 0.576 & 1.05 & 0.772 & 20.08 & $23.80^{*}$ & 22.22 \\
\hline & \pm 0.35 & \pm 0.95 & \pm 0.61 & \pm 0.21 & \pm 0.57 & \pm 0.46 & \pm 0.10 & \pm 0.28 & \pm 0.15 & \pm 0.43 & \pm 0.73 & \pm 1.10 \\
\hline \multirow[t]{2}{*}{$30^{\prime} \mathrm{RP}$} & 15.14 & $18.43^{*}$ & $17.98^{*}$ & 4.47 & 4.43 & $6.83^{*}$ & 0.758 & 0.488 & 1.07 & 20.37 & 24.04 & $25.89^{*}$ \\
\hline & \pm 0.86 & \pm 0.17 & \pm 0.94 & \pm 0.71 & \pm 0.48 & \pm 0.43 & \pm 0.22 & \pm 0.11 & \pm 0.15 & \pm 1.68 & \pm 0.27 & \pm 1.30 \\
\hline \multirow[t]{2}{*}{$60^{\prime} \mathrm{RP}$} & 14.51 & $18.17^{*}$ & 15.45 & 3.63 & $4.86^{*}$ & $4.82^{*}$ & 0.266 & 0.425 & $0.473^{*}$ & 18.40 & $23.49^{*}$ & 20.74 \\
\hline & \pm 1.04 & \pm 0.97 & \pm 0.43 & \pm 0.22 & \pm 0.28 & \pm 0.26 & \pm 0.04 & \pm 0.11 & \pm 0.05 & \pm 1.20 & \pm 0.52 & \pm 0.61 \\
\hline
\end{tabular}

Data expressed as $\mu \mathrm{mol} / \mathrm{g}$ dry wt, mean $\pm \mathrm{SE}$ for control (C), $100 \mu \mathrm{M}$ allopurinol-treated (ALLO) hearts and $100 \mu \mathrm{M}$ hypoxanthine-treated (HYPO) hearts. Equil, equilibration; RP, reperfusion; TAN, ATP + ADP + AMP.

${ }^{*} P<0.05$ vs. control. Each mean represents a minimum of six hearts.

Myocardial ischemia results in the rapid breakdown of ATP to ADP and AMP (18-20), which is further catabolized to adenosine, inosine, hypoxanthine, and uric acid (21-23). Xanthine dehydrogenase, the enzyme that catalyzes the oxidation of hypoxanthine and xanthine to uric acid, is believed to be converted rapidly to xanthine oxidase during ischemia $(9,12)$. During reperfusion, there is a rapid washout of the salvageable purines, and the action of xanthine oxidase, in the presence of high levels of hypoxanthine and oxygen, is thought to be the primary source of oxygen free radicals.

Previous studies using allopurinol, a competitive inhibitor of xanthine oxidase, have shown it to have a beneficial effect on ischemic myocardium (14-16). DeWall et al. (16) showed that treatment with allopurinol improved cardiac output and myocardial contractility and inhibited the release of uric acid following occlusion of the left anterior descending coronary artery in the dog. Parker and Smith (15) reported that pretreatment with allopurinol improved the survival rate and greatly reduced the release of uric acid in dogs subjected to 10 min of cardiac arrest after electrically induced fibrillation.

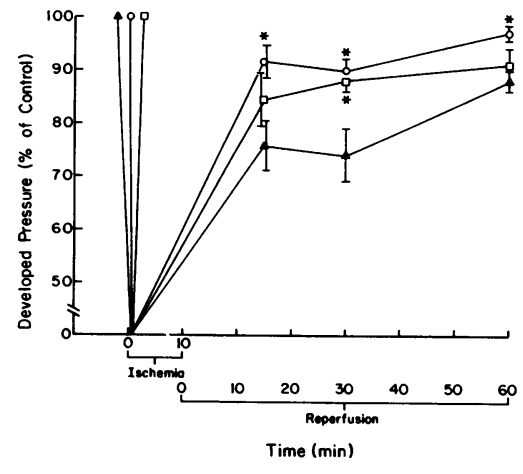

Figure 1. Effect of 100 $\mu \mathrm{M}$ allopurinol and 100 $\mu \mathrm{M}$ hypoxanthine on recovery of left ventricular function after global ischemia. Recovery of function is expressed as the percent of the developed pressure at the end of 30 min of equilibration. Developed pressure at the end of $30 \mathrm{~min}$ of equilibration was not

different among the three groups $(94 \pm 3,90 \pm 4,87 \pm 3 \mathrm{mmHg}$ for control ( $\triangle$ ), allopurinol (O), and hypoxanthine ( $\square$ ) groups, respectively). Each point represents a mean \pm SE for six hearts. *Significantly different from untreated control group $(P<0.05)$.
Lindsay et al. (14) reported a greater preservation of ATP levels in dogs pretreated with allopurinol after $60 \mathrm{~min}$ of global, normothermic ischemia. These reports suggested that allopurinol preserved the pool of purine bases for the resynthesis of adenine nucleotides via the purine salvage mechanism.

Much of the recent interest in allopurinol has focused on its ability to prevent the formation of oxygen-derived free radicals (6-8). Manning et al. (6) showed that pretreatment with allopurinol provided protection from ischemia-induced arrhythmias in rats during occlusion of the left anterior descend-

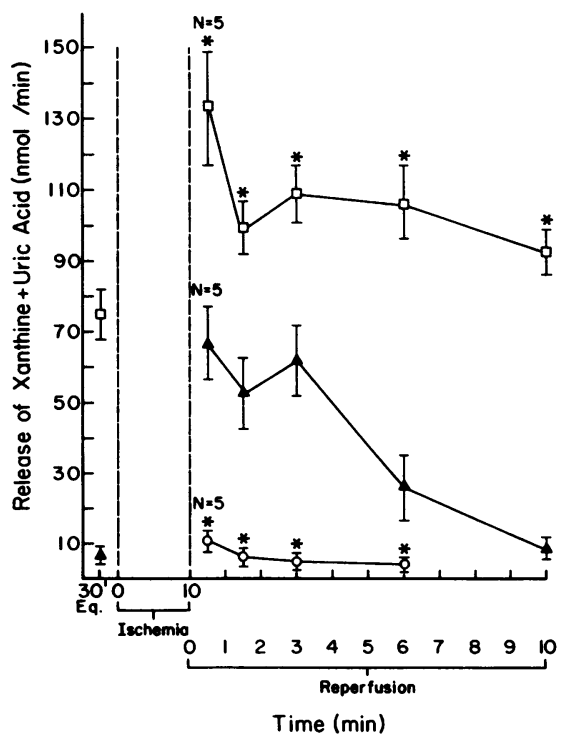

Figure 2. Effect of allopurinol (o) and hypoxanthine ( $\square$ ) on the release of xanthine and uric acid during reperfusion. Timed collections of coronary effluent were taken at 30 and $90 \mathrm{~s}$, and at 3, 6, and 10 min of reperfusion. Samples were dried, resuspended in $\mathrm{KH}_{2} \mathrm{PO}_{4}$ and analyzed by HPLC. Each point represents a mean \pm SE for five hearts. *Significantly different from untreated control $(\Lambda)(P<0.01)$. 


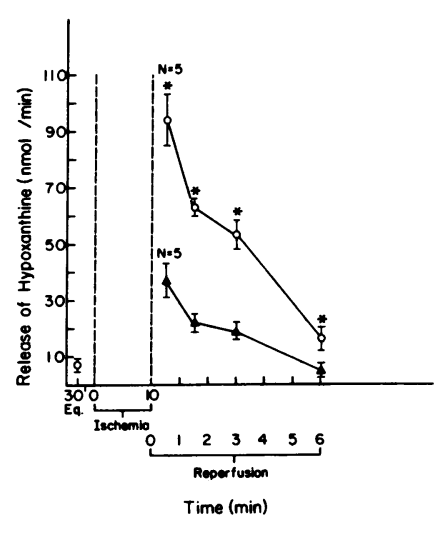

Figure 3. Effect of allopurinol (o) on the release of hypoxanthine during reperfusion. *Significantly different from untreated control $(\Lambda)(P<0.02)$.

ing coronary artery and an even greater reduction in arrhythmias during reperfusion. They suggested that, by inhibiting xanthine oxidase, allopurinol prevented the formation of superoxide radicals and thus protected the myocardium from ischemic injury. Chambers et al. (7), using a canine model of coronary artery occlusion and reperfusion, reported that allopurinol pretreatment diminished the region of infarction to the same extent as that of superoxide dismutase, a free radical scavenger. They concluded that xanthine oxidase was a source of oxygen free radicals and that allopurinol protected the ischemic myocardium by preventing the formation of free radicals. Stewart et al. (8) reported similar findings in dogs subjected to $60 \mathrm{~min}$ of global, hypothermic ischemia and $45 \mathrm{~min}$ of reperfusion. Dogs pretreated for $72 \mathrm{~h}$ with allopurinol exhibited the same degree of recovery of ventricular function as those that received superoxide dismutase and mannitol. The authors concluded that allopurinol's beneficial effects were due to the inhibition of the xanthine oxidase reaction.

The results of these studies (6-8) suggest that by inhibiting xanthine oxidase during reperfusion, allopurinol prevents superoxide radical-induced tissue injury. The evidence for this mechanism of action is based on the observation that allopurinol pretreatment produces the same degree of functional recovery from ischemia as that seen with superoxide dismutase, a free radical scavenger. None of these studies measured the release of hypoxanthine, xanthine, or uric acid, nor was adenine nucleotide content measured to determine whether there was any effect on the repletion of high energy phosphates. In addition, there is currently no technology available to directly measure oxygen free radicals or their source.

If allopurinol enhances repletion of ATP by preserving substrate, then one might expect to see similar results if hypoxanthine is supplied to the myocardium. Minaga et al. (24) reported an increased uptake of labeled hypoxanthine and its incorporation into the adenine nucleotide pool in allopurinoltreated myocardium. The results of this study show that hypoxanthine treatment augmented myocardial adenine nucleotide levels during reperfusion, although not to the same extent as did allopurinol treatment. The hypoxanthine-treated hearts showed greater recovery of ventricular function than did control hearts, although only at $30 \mathrm{~min}$ of reperfusion was the difference significant. The difference in functional recovery between the allopurinol and hypoxanthine treatments may be due to the fact that a significant amount of the hypoxanthine was degraded to xanthine and uric acid.

The results of hypoxanthine treatment in this study do not support the concept of free radical-induced reperfusion injury. Hearts treated with hypoxanthine exhibited a greater release of xanthine and uric acid during reperfusion than did untreated hearts, yet they demonstrated a slightly greater recovery of ventricular function. In addition, there was not an increased incidence of ventricular arrhythmias during reperfusion in the hypoxanthine-treated hearts compared to the control or allopurinol-treated hearts. If hypoxanthine metabolism results in the formation of oxygen free radicals, which in turn cause significant reperfusion injury, one would expect the hypoxanthine-treated hearts to have shown a diminished rather than improved functional recovery compared with the untreated hearts.

There is other evidence to suggest that allopurinol's mechanism of action may differ from that of the free radical scavengers, superoxide dismutase and catalase. Allopurinol is a competitive inhibitor of xanthine oxidase, while alloxanthine, a metabolite of allopurinol, is a noncompetitive inhibitor (25). This difference in the active form of the drug may explain why pretreatment with allopurinol produces the greatest degree of protection of ischemic myocardium, whereas the beneficial effects of superoxide dismutase can be observed even when presented only during reperfusion. The results of this study show that allopurinol treatment preserved the total adenine nucleotide pool during the ischemic period, a finding that has not been previously documented in ischemic myocardium, although Lindsay et al. (14) did report higher ATP levels after global ischemia in canines pretreated for $72 \mathrm{~h}$ with allopurinol. Cunningham et al. (2) reported a conservation of ATP and total adenine nucleotide content in ischemic kidneys following allopurinol treatment. Fox and Marchant (27) observed that allopurinol ribonucleotide, a metabolite of allopurinol, inhibits 5 -nucleotidase. These results may explain the preservation of ADP and AMP levels during ischemia in the allopurinol-treated hearts seen in this study. Superoxide dismutase and catalase, which catalyze the conversion of oxygen free radicals to $\mathrm{H}_{2} \mathrm{O}$ and $\mathrm{O}_{2}$, would not be expected to maintain the adenine nucleotide pool during the ischemic period.

Allopurinol, with a molecular weight of 137 , can easily traverse the endothelium, whereas superoxide dismutase and catalase, with molecular weights of 32,000 and 250,000 , respectively, would not be expected to pass through membranes.

While not totally mimicking the in vivo situation, the isolated rat heart preparation has proved useful in the study of myocardial ischemia and reperfusion injury. In addition, the use of an asanguinous preparation circumvents the production of oxygen-derived free radicals by neutrophils.

The results of the present study confirm that allopurinol has a protective effect on ischemic myocardium, both in terms of functional recovery and in the repletion of high energy phosphates. The fact that allopurinol maintains the total adenine nucleotide pool during ischemia suggests that its beneficial effects may be due to mechanisms other than the inhibition of oxygen free radical production.

\section{Acknowledgments}

Supported by a grant from the American Heart Association, Virginia Affiliate. Dr. Robert M. Mentzer, Jr. is the recipient of Research Career Development Award HL-01299 from the National Institutes of Health. 


\section{References}

1. Hearse, D. J. 1979. Oxygen deprivation and early myocardial contractile failure: a reassessment of the possible role of adenosine triphosphate. Am. J. Cardiol. 44:1115-1121.

2. DeBoer, L. W. V., J. S. Ingwall, R. A. Kloner, and E. Branwald. 1980. Prolonged derangements of canine myocardial purine metabolism after a brief coronary artery occlusion not associated with anatomic evidence of necrosis. Proc. Natl. Acad. Sci. USA. 77:5471-5475.

3. Pasque, M. K., T. L. Spray, G. L. Pellom, van P. Trigt, R. B. Peyton, W. D. Currie, and A. S. Wechsler. 1982. Ribose-enhanced myocardial recovery following ischemia in the isolated working rat heart. J. Thorac. Cardiovasc. Surg. 83:390-398.

4. Reibel, D. K., and M. J. Rovetto. 1978. Myocardial ATP synthesis and mechanical function following oxygen deficiency. Am. J. Physiol. 234 (Heart Circ. Physiol. 3):H620-H624.

5. Ward, H. B., J. B. Kriett, S. Einzig, R. W. Bianco, R. W. Anderson, and J. E. Foker. 1983. Adenine nucleotides and cardiac function following global myocardial ischemia. Surg. Forum. 34:264-266. (Abstr.)

6. Manning, A. S., D. J. Coltart, and D. J. Hearse. 1984. Ischemia and reperfusion-induced arrhythmias in the rat. Effects of xanthine oxidase inhibition with allopurinol. Circ. Res. 55:545-548.

7. Chambers, D. E., D. A. Parks, G. Patterson, R. Roy, J. M. McCord, S. Yosida, L. F. Parmley, and J. M. Downey. 1985. Xanthine oxidase as a source of free radical damage in myocardial ischemia. $J$. Mol. Cell Cardiol. 17:145-152.

8. Stewart, J. R., S. L. Crute, V. Loughlin, M. L. Hess, and L. J. Greenfield. 1985. Prevention of free radical-induced myocardial reperfusion injury with allopurinol. J. Thorac. Cardiovasc. Surg. 90:6872.

9. McCord, J. M. 1984. Are free radicals a major culprit? In Therapeutic Approaches to Myocardial Infarct Size Limitation. D. J: Hearse and D. M. Yellon, editors. Raven Press, New York. 209-218.

10. Bernier, M., D. J. Hearse, and A. S. Manning. 1986. Reperfusion-induced arrhythmias and oxygen-derived free radicals. Studies with "anti-free radical" interventions and a free radical-generating system in the isolated perfused rat heart. Circ. Res. 58:331-340.

11. Hearse, D. J., A. S. Manning, J. M. Downey, and D. M. Yellon. 1986. Xanthine oxidase: a critical mediator of myocardial injury during ischemia and reperfusion? Acta Physiol. Scand. 548(Suppl.):65-78.

12. Werns, S. W., M. J. Shea, and B. R. Lucchesi. 1986. Free radicals and myocardial injury: pharmacologic implications. Circulation. 74:1-5.

13. Arnold, W. L., R. A. DeWall, P. Kezdi, and H. H. J. Zwarti.
1980. The effect of allopurinol on the degree of early myocardial ischemia. Am. Heart J. 99:614-624.

14. Lindsay, W. G., L. H. Toledo-Pereyra, J. E. Foker, and R. L. Varco. 1975. Metabolic myocardial protection with allopurinol during cardiopulmonary bypass and aortic cross-clamping. Surg. Forum. 26:259-260. (Abstr.)

15. Parker, J. C., and E. E. Smith. 1972. Effects of xanthine oxidase inhibition in cardiac arrest. Surgery. 71:339-344.

16. DeWall, R. A., K. A. Vasko, E. L. Stanley, and P. Kezdi. 1971 Responses of the ischemic myocardium to allopurinol. Am. Heart J. 82:362-370.

17. Parker, J. C., E. E. Smith, and C. E. Jones. 1976. The role of nucleoside and nucleobase metabolism in myocardial adenine nucleotide regeneration after cardiac arrest. Circ. Shock. 3:11-20.

18. Jennings, R. B., K. A. Reimer, M. L. Hill, and S. E. Mayer. 1981. Total ischemia in dog hearts, in vitro: 1. Comparison of high energy phosphate production, utilization, and depletion, and of adenine nucleotide catabolism in total ischemia in vitro vs. severe ischemia in vivo. Circ. Res. 49:892-900.

19. Neely, J. R., M. J. Rovetto, J. T. Whitmer, and H. E. Morgan. 1973. Effects of ischemia on ventricular function and metabolism in the isolated working rat heart. Am. J. Physiol. 225:651-658.

20. Berne, R. M. 1974. Cardiac nucleotides in hypoxia: possible role in regulation of coronary blood flow. Am. J. Physiol. 2p4:317322.

21. Jennings, R. B., J. Schaper, M. L. Hill, C. Steenberger, Jr., and K. A. Reimer. 1985. Effect of reperfusion late in the phase of reversible ischemic injury. Changes in cell volume, electrolytes, metabolites, and ultrastructure. Circ. Res. 56:262-278.

22. Berne, R. M., and R. Rubio. 1974. Adenine nucleotide metabolism in the heart. Circ. Res. 34, 35, (Suppl. 3):109-120.

23. Imai, S., A. L. Riley, and R. M. Berne. 1964. Effect of ischemia on adenine nucleotides in cardiac and skeletal muscle. Circ. Res. 15:443-450.

24. Minaga, T., K. Takeda, T. Nakamura, A. Kizu, and H. Ichiji. 1978. Effect of xanthine oxidase inhibitor on myocardial ischemia. In Heart Function and Metabolism. T. Kobayashi, T. Sano, and N. S. Dhalla, editors. University Park, Baltimore. Vol II. 555-558.

25. Elion, G. B. 1966. Enzymatic and metabolic studies with allopurinol. Ann. Rheum. Dis. 25:608-614.

26. Cunningham, S. K., T. V. Keaveny, and P. Fitzgerald. 1974. Effect of allopurinol on tissue ATP, ADP and AMP concentrations in renal ischemia. Br. J. Surg. 61:562-565.

27. Fox, I., and P. Marchant. 1976. Purine catabolism in man: inhibition of 5 ' phosphomonoesterase activities from placental microsomes. Can. J. Biochem. 54:1055-1060. 